\title{
Beyond the Corey Reaction: One-Step Diolefination of Cyclic Ketones
}

\author{
Ekaterina D. Butova, ${ }^{a}$ Andrey A. Fokin, ${ }^{a, b *}$ and Peter R. Schreiner ${ }^{a *}$
}

\section{CONTENTS}

1. The XYZ-coordinates of computed species (Table 1S) S2

2. The MP2/cc-pVDZ optimized geometries of the transition structures (Figure S1) S5

3. The ${ }^{13} \mathrm{C}-\mathrm{NMR}$ spectra of new compounds $\quad$ S6 
Table 1s. The XYZ-coordinates of computed species:

TS1 (B3PW91/ 6-311+G(d), HF= -941.51391)

$\begin{array}{llll}\text { C } & 2.701490 & 1.462035 & 0.076425\end{array}$

$\begin{array}{llll}\text { C } & 3.722614 & 0.486330 & -0.513136\end{array}$

$\begin{array}{llll}\text { C } & 3.579688 & -0.977085 & -0.094427\end{array}$

$\begin{array}{llll}\text { C } & 2.194875 & -1.608247 & -0.284499\end{array}$

$\begin{array}{llll}\text { C } & 1.147094 & -1.096627 & 0.707990\end{array}$

$\begin{array}{llll}\text { C } & 0.457700 & 0.260854 & 0.427269\end{array}$

$\begin{array}{llll}\text { C } & 1.275713 & 1.234861 & -0.444750\end{array}$

$\begin{array}{llll}\mathrm{H} & 3.014253 & 2.479100 & -0.187881\end{array}$

$\begin{array}{llll}\mathrm{H} & 2.708507 & 1.425065 & 1.172370\end{array}$

$\mathrm{H} \quad 0.717371 \quad 2.176756 \quad-0.444484$

$\begin{array}{llll}\mathrm{H} & 1.342580 & 0.901960 & -1.487806\end{array}$

H $\quad 3.666536 \quad 0.551867 \quad-1.609292$

$\mathrm{H} \quad 4.734120 \quad 0.820411 \quad-0.249340$

$\mathrm{H} \quad 4.319658 \quad-1.565802 \quad-0.651391$

$\begin{array}{llll}\mathrm{H} & 3.856276 & -1.078317 & 0.964045\end{array}$

$\begin{array}{llll}\mathrm{H} & 0.370643 & -1.851990 & 0.870122\end{array}$

$\mathrm{H} \quad 1.621231 \quad-0.967686 \quad 1.688280$

$\mathrm{H} \quad 1.857464-1.475863 \quad-1.321830$

H $\quad 2.300928 \quad-2.691663 \quad-0.149447$

$\begin{array}{llll}\text { O } & -0.099429 & 0.781430 & 1.459958\end{array}$

S $\quad-2.466551-0.137894-0.329342$

$\begin{array}{llll}\text { O } & -3.608872 & -0.562046 & -1.165086\end{array}$

$\begin{array}{llll}\text { C } & -2.576296 & 1.574819 & 0.142642\end{array}$

$\begin{array}{llll}\text { C } & -2.456563 & -0.982667 & 1.239226\end{array}$

$\begin{array}{llll}\text { C } & -0.841706 & -0.278729 & -0.907857\end{array}$

$\mathrm{H} \quad-2.518584 \quad 2.164676 \quad-0.772639$

$\begin{array}{llll}\mathrm{H} & -2.303948 & -2.042902 & 1.036997\end{array}$

$\begin{array}{llll}\mathrm{H} & -3.538153 & 1.726492 & 0.632761\end{array}$

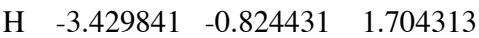

$\mathrm{H} \quad-1.711933 \quad 1.743974 \quad 0.806722$

$\begin{array}{llll}\mathrm{H} & -1.625832 & -0.536216 & 1.807008\end{array}$

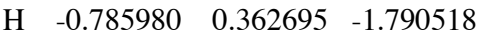

H $\quad-0.691718 \quad-1.320161 \quad-1.200777$
TS2 (B3PW91/ 6-311+G(d), HF= -1059.4120818)

$\begin{array}{llll}\text { C } & 3.595138 & -.059775 & -.941209\end{array}$

$\begin{array}{llll}\text { C } & 1.652608 & -1.491661 & .826700\end{array}$

$\begin{array}{llll}\text { C } & 2.789374 & -2.284244 & .153934\end{array}$

$\begin{array}{llll}\text { C } & 3.969008 & -1.442153 & -.374661\end{array}$

$\begin{array}{llll}\text { C } & .321507 & 1.389214 & -.374785\end{array}$

$\begin{array}{llll}\text { C } & 3.755997 & 1.084096 & .076951\end{array}$

$\begin{array}{llll}\text { C } & 2.794698 & 2.268287 & -.064628\end{array}$

$\begin{array}{llll}\text { C } & 1.381697 & 2.079180 & .516051\end{array}$

$\begin{array}{llll}\mathrm{H} & 2.365039 & -2.866082 & -.675033\end{array}$

$\begin{array}{llll}\mathrm{H} & 2.034482 & -.559176 & 1.242263\end{array}$

H $\quad 1.271186 \quad-2.039852 \quad 1.692468$

$\begin{array}{llll}\mathrm{H} & -.501788 & 2.098760 & -.507989\end{array}$

$\begin{array}{llll}\mathrm{H} & .714886 & 1.205562 & -1.382824\end{array}$

$\begin{array}{llll}\mathrm{H} & 1.003455 & 3.077040 & .762973\end{array}$

$\begin{array}{llll}\mathrm{H} & 1.433656 & 1.564073 & 1.480469\end{array}$

H $\quad 2.728450 \quad 2.576143 \quad-1.118007$

$\begin{array}{llll}\mathrm{H} & 3.246952 & 3.121635 & .456155\end{array}$

$\begin{array}{llll}\mathrm{H} & 3.664895 & .690988 & 1.096489\end{array}$

$\begin{array}{llll}\mathrm{H} & 4.787348 & 1.454571 & .013763\end{array}$

H $\quad 2.572778 \quad-.081828 \quad-1.325305$

$\mathrm{H} \quad 4.497523 \quad-2.031314 \quad-1.133070$

$\begin{array}{llll}\mathrm{H} & 4.697785 & -1.294882 & .433039\end{array}$

$\begin{array}{llll}\mathrm{H} & 3.189398 & -3.030513 & .850242\end{array}$

$\begin{array}{llll}\text { C } & .471177 & -1.208540 & -.118372\end{array}$

$\mathrm{H} \quad-.219773 \quad-2.058430 \quad-.054183$

H $\quad .810491 \quad-1.183168-1.161463$

$\begin{array}{llll}\mathrm{H} & 4.219470 & .163515 & -1.814205\end{array}$

$\begin{array}{llll}\text { C } & -.308544 & .086487 & .204067\end{array}$

$\begin{array}{llll}\mathrm{O} & -.742273 & .182831 & 1.415067\end{array}$

$\begin{array}{llll}\mathrm{S} & -3.260847 & -.112707 & -.334467\end{array}$

$\begin{array}{llll}\mathrm{O} & -4.471080 & -.252366 & -1.168675\end{array}$

$\begin{array}{llll}\text { C } & -3.275199 & 1.386599 & .624368\end{array}$

$\begin{array}{llll}\text { C } & -3.149783 & -1.383142 & .906750\end{array}$

$\begin{array}{llll}\text { C } & -1.688215 & -.122216 & -1.071932\end{array}$

$\begin{array}{llll}\mathrm{H} & -3.272061 & 2.220247 & -.078150\end{array}$

$\begin{array}{llll}\mathrm{H} & -3.065321 & -2.337307 & .386068\end{array}$

$\mathrm{H} \quad-4.190516 \quad 1.393231 \quad 1.216675$

H $\quad-4.065971 \quad-1.352581 \quad 1.496892$

$\begin{array}{llll}\mathrm{H} & -2.357010 & 1.340975 & 1.234998\end{array}$

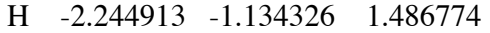

$\mathrm{H} \quad-1.689496 \quad .688246 \quad-1.804466$

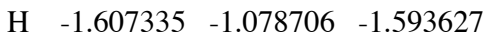


TS3 (B3PW91/ 6-311+G(d), HF= -1138.0302252)

$\begin{array}{lrrr}\mathrm{C} & 4.683114 & -1.159785 & -0.273653 \\ \mathrm{C} & 1.338519 & -1.498623 & 0.449016 \\ \mathrm{C} & 2.338934 & -2.344696 & -0.340074 \\ \mathrm{C} & 3.781859 & -2.318907 & 0.184767 \\ \mathrm{C} & -0.231251 & 1.145510 & -0.459200 \\ \mathrm{C} & 3.101987 & 2.461657 & 0.057529 \\ \mathrm{C} & 1.648348 & 2.901986 & -0.149414 \\ \mathrm{C} & 0.625378 & 1.958870 & 0.523677 \\ \mathrm{C} & 4.373439 & 0.244686 & 0.273825 \\ \mathrm{C} & 3.469737 & 1.117962 & -0.600267 \\ \mathrm{H} & 2.339661 & -2.023019 & -1.391197 \\ \mathrm{H} & 1.742579 & -0.496082 & 0.577938 \\ \mathrm{H} & 1.227805 & -1.895334 & 1.464000 \\ \mathrm{H} & -1.024774 & 1.805632 & -0.828234 \\ \mathrm{H} & 0.354410 & 0.878003 & -1.348113 \\ \mathrm{H} & -0.047423 & 2.529549 & 1.170147 \\ \mathrm{H} & 1.143246 & 1.286979 & 1.212724 \\ \mathrm{H} & 1.438094 & 2.986160 & -1.224324 \\ \mathrm{H} & 1.537380 & 3.917464 & 0.248967 \\ \mathrm{H} & 3.279295 & 2.393710 & 1.139182 \\ \mathrm{H} & 3.779834 & 3.247264 & -0.298483 \\ \mathrm{H} & 2.559810 & 0.566924 & -0.851074 \\ \mathrm{H} & 3.965840 & 1.298214 & -1.562584 \\ \mathrm{H} & 5.316186 & 0.785183 & 0.430382 \\ \mathrm{H} & 3.927931 & 0.152800 & 1.273429 \\ \mathrm{H} & 4.696701 & -1.129699 & -1.372306 \\ \mathrm{H} & 5.706766 & -1.424094 & 0.018666 \\ \mathrm{H} & 4.275165 & -3.246812 & -0.130928 \\ \mathrm{H} & 3.772062 & -2.354618 & 1.283153 \\ \mathrm{H} & 1.983427 & -3.383744 & -0.356111 \\ \mathrm{C} & -0.038988 & -1.418547 & -0.222314 \\ \mathrm{H} & -0.629644 & -2.301197 & 0.051391 \\ \mathrm{H} & 0.088332 & -1.451249 & -1.313293 \\ \mathrm{C} & -0.841290 & -0.152149 & 0.160112 \\ \mathrm{O} & -1.217221 & -0.064159 & 1.386264 \\ \mathrm{~S} & -3.828892 & -0.163185 & -0.280331 \\ \mathrm{O} & -5.081243 & -0.224545 & -1.060821 \\ \mathrm{C} & -3.647117 & 1.408890 & 0.538716 \\ \mathrm{C} & -3.806927 & -1.320139 & 1.070007 \\ \mathrm{C} & -2.295697 & -0.436839 & -1.043248 \\ \mathrm{H} & -3.553000 & 2.171527 & -0.234295 \\ \mathrm{H} & -3.866115 & -2.319531 & 0.637912 \\ \mathrm{H} & -4.548927 & 1.577912 & 1.127642 \\ \mathrm{H} & -4.677632 & -1.123862 & 1.695994 \\ & -2.739364 & 1.312833 & 1.153085\end{array}$

TS4 (MP2/cc-pVDZ, MP2 $=-863.1251262)$

$\begin{array}{llll}\text { C } & 3.136888 & -.776797 & .080857\end{array}$

$\begin{array}{llll}\text { C } & 3.206026 & .595604 & -.602956\end{array}$

$\begin{array}{llll}\text { C } & 1.879416 & 1.367189 & -.557335\end{array}$

$\begin{array}{llll}\text { C } & 1.206241 & 1.328466 & .824474\end{array}$

$\begin{array}{llll}\text { C } & .403787 & .062778 & 1.100521\end{array}$

$\begin{array}{llll}\text { C } & .627031 & -1.161244 & .453684\end{array}$

C $\quad \begin{array}{llll}\text { C } & 1.881238 & -1.579821 & -.295479\end{array}$

$\mathrm{H} \quad 1.977123 \quad 1.467726 \quad 1.608272$

$\begin{array}{llll}\mathrm{H} & .524235 & 2.193109 & .930505\end{array}$

$\mathrm{H} \quad 2.063411 \quad 2.415178 \quad-.857497$

$\begin{array}{llll}\mathrm{H} & 1.181259 & .946491 & -1.305275\end{array}$

$\begin{array}{llll}\mathrm{H} & 3.995518 & 1.200979 & -.119201\end{array}$

$\begin{array}{llll}\mathrm{H} & 3.511517 & .466653 & -1.658322\end{array}$

$\mathrm{H} \quad 4.041214 \quad-1.353512 \quad-.186352$

$\mathrm{H} \quad 3.157432 \quad-.655674 \quad 1.179381$

H $\quad 2.060206 \quad-2.649173 \quad-.081956$

H $\quad \begin{array}{llll}1.729751 & -1.521830 & -1.393343\end{array}$

$\begin{array}{llll}\mathrm{H} & .032283 & -1.996780 & .847822\end{array}$

$\begin{array}{llll}\text { C } & -.870626 & .202142 & 1.714658\end{array}$

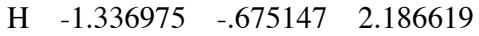

$\mathrm{H} \quad-1.156658 \quad 1.163269 \quad 2.168102$

S $\quad-2.138039 \quad-.127249 \quad-.167141$

$\begin{array}{llll}\mathrm{O} & -3.511565 & -.655728 & .179196\end{array}$

$\begin{array}{llll}\text { C } & -2.176531 & 1.527221 & -.875550\end{array}$

$\begin{array}{llll}\text { C } & -1.134707 & -1.010571 & -1.164206\end{array}$

$\begin{array}{llll}\mathrm{H} & -2.787600 & 1.514607 & -1.790679\end{array}$

$\mathrm{H} \quad-2.621903 \quad 2.186704 \quad-.115083$

$\mathrm{H} \quad-1.138410 \quad 1.826809 \quad-1.087411$

$\mathrm{H} \quad-1.376873 \quad-2.071394 \quad-1.256689$

$\mathrm{H} \quad-.506966 \quad-.506012-1.900220$ 
TS5 (MP2/cc-pVDZ, MP2 $=-863.1947528)$

$\begin{array}{llll}\text { C } & -1.832911 & -1.784393 & -.240245\end{array}$

$\begin{array}{llll}\text { C } & -2.514182 & -.674259 & -1.055683\end{array}$

$\begin{array}{llll}\text { C } & -3.216648 & .406822 & -.218817\end{array}$

$\begin{array}{llll}\text { C } & -2.256372 & 1.319588 & .609643\end{array}$

$\begin{array}{llll}\text { C } & -.811981 & 1.184646 & .173163\end{array}$

$\begin{array}{llll}\text { C } & -.108953 & -.072431 & .618030\end{array}$

$\begin{array}{llll}\text { C } & -1.009758 & -1.266198 & .950128\end{array}$

$\begin{array}{llll}\mathrm{H} & -2.567175 & 2.372685 & .499850\end{array}$

$\mathrm{H} \quad-2.333312 \quad 1.075988 \quad 1.683688$

$\begin{array}{llll}\mathrm{H} & -3.790085 & 1.046554 & -.913316\end{array}$

$\begin{array}{llll}\mathrm{H} & -3.958413 & -.063683 & .453593\end{array}$

$\mathrm{H} \quad-1.765367 \quad-.181707 \quad-1.701444$

H $\quad-3.252765-1.134258-1.737521$

$\begin{array}{llll}\mathrm{H} & -2.596756 & -2.488841 & .140144\end{array}$

$\begin{array}{llll}\mathrm{H} & -1.173199 & -2.361787 & -.913952\end{array}$

$\mathrm{H} \quad-.378519 \quad-2.088008 \quad 1.335794$

$\begin{array}{llll}\mathrm{H} & -1.692506 & -.995393 & 1.778759\end{array}$

$\begin{array}{llll}\mathrm{H} & .529433 & -.464283 & -.489835\end{array}$

$\begin{array}{llll}\text { C } & -.219149 & 2.126713 & -.599251\end{array}$

$\mathrm{H} \quad .782473 \quad 1.977023 \quad-1.011399$

$\begin{array}{llll}\mathrm{H} & -.745065 & 3.049938 & -.864518\end{array}$

S $\quad 2.639068 \quad-.675474 \quad-.001152$

$\begin{array}{llll}\text { O } & 1.543605 & -.829684 & -1.140946\end{array}$

$\begin{array}{llll}\text { C } & 3.406310 & .937203 & -.315587\end{array}$

$\begin{array}{llll}\text { C } & 1.059798 & .075397 & 1.432155\end{array}$

$\begin{array}{llll}\mathrm{H} & 3.851565 & .923429 & -1.321870\end{array}$

$\mathrm{H} \quad 2.666555 \quad 1.747108 \quad-.234446$

$\begin{array}{llll}\mathrm{H} & 4.197707 & 1.070411 & .441713\end{array}$

$\mathrm{H} \quad 1.237060 \quad-.646352 \quad 2.242231$

$\begin{array}{llll}\mathrm{H} & 1.437521 & 1.087713 & 1.637301\end{array}$

TS4_Li (MP2/cc-pVDZ, MP2=--870.4592798) C $-0.8287779-0.0129666-3.2829739$

C $0.38839050 .9192294-3.3471845$

C $1.21930040 .9318109-2.0558922$

C $1.4775798-0.4738915-1.4892255$

C $0.3284160-1.0430550-0.6707815$

C - $1.0163456-0.6654797-0.8222307$

C - $1.62224260 .1113806-1.9701621$

H $1.7016864-1.1722068-2.3177904$

H $2.3840316-0.4767984-0.8565935$

H $2.18304421 .4337164-2.2515242$

H $0.70333011 .5419675-1.2891050$

H $1.03230140 .6113414-4.1904577$

H $0.05502811 .9496473-3.5668200$

H - $1.49928770 .2095930-4.1308733$

H $-0.5170732-1.0659814-3.4076537$

H -2.6490850 -0.2669728 -2.1164991

$\mathrm{H}-1.73757741 .1827736-1.7102425$

H - $1.7233949-1.2660459-0.2325034$

C $0.6159797-1.81702350 .4764244$

H - $0.1662797-2.44678950 .9195754$

H $1.6491814-2.10390760 .7164702$

S $0.0662699-0.00090051 .9505709$

O $-0.2212156-0.24236363 .4778037$

C 1.50534991 .07057891 .8126856

C - 1.16659110 .87015891 .1972540

H 1.29117412 .03425422 .2984035

H 2.32617950 .54476352 .3227191

H 1.72691151 .19989690 .7428433

H -2.1752195 0.44591831 .2404678

H -0.94377621 .72084080 .5497769$

Li - 1.55754270 .96059983 .8689326
TS5_Li (MP2/cc-pVDZ, MP2=--870.51832)

C $-1.74595990 .5011153-1.651836$

C $-1.1368196-0.8461356-2.0868392$

C $0.0436919-0.7272012-3.0659856$

C $1.24806590 .0919105-2.5283389$

C $1.39859850 .0347105-1.0205676$

C $0.38528970 .8361739-0.2219323$

C $-0.74536931 .4991543-1.0199466$

H $2.1709552-0.2926982-2.9919050$

H $1.16108261 .1434735-2.8478322$

H $0.3828951-1.7517536-3.2974186$

H $-0.3145493-0.2900614-4.0144781$

H $-0.7331630-1.4166484-1.2184124$

H - $1.9218939-1.4713697-2.5558643$

H - $2.23281180 .9638207-2.5279652$

H - $2.59762150 .3486478-0.9442643$

H - $1.29931802 .1879842-0.3590731$

H - $0.31240592 .1308637-1.8128369$

H $-0.2532605-0.13805320 .3760741$

C $2.3700545-0.7125357-0.4456141$

H $2.4968554-0.77738860 .6393878$

H $3.0935176-1.2560347-1.0603529$

S - 0.49650690 .16844522 .5499193

O $-0.9233758-0.75706371 .2757750$

C $0.8534816-0.79064393 .2682215$

C 0.76260621 .46246910 .9951397

H $0.4460744-1.66209663 .8010665$

H $1.5447723-1.10825172 .4751548$

H $1.3674272-0.12268933 .9798122$

H 0.28391082 .40096841 .2984748

H 1.76710101 .29770971 .4075595

Li -2.2311903-1.3732295 0.1606942 


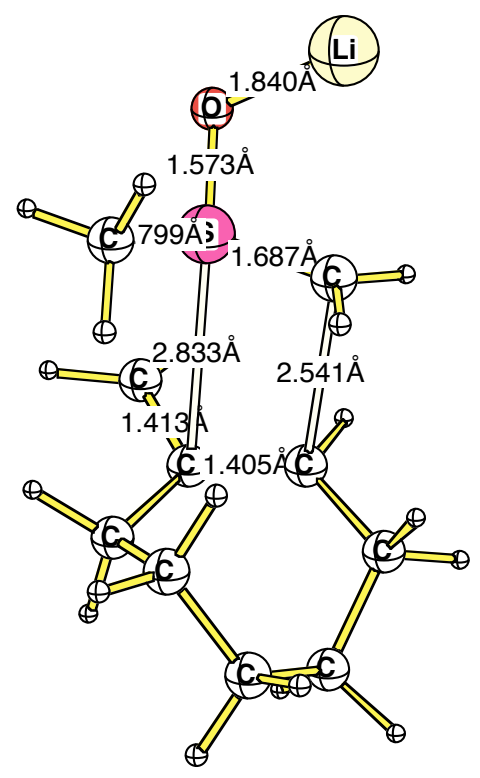

TS4 Li

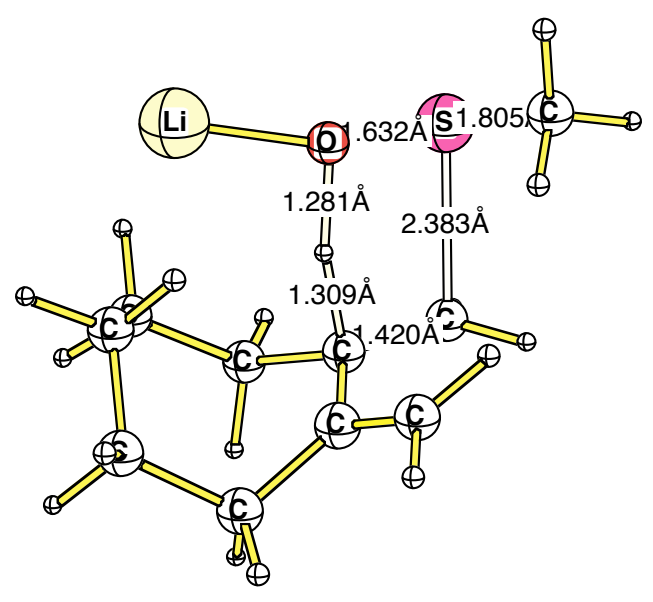

TS5_Li

Figure 1s. The MP2/cc-pVDZ optimized geometries of the transition structures for the [2,3]sigmatropic rearrangement of ylide and the elimination of sulfenic acid from sulfoxid with Li-ion present. 


\section{${ }^{13} \mathrm{C}$ NMR spectra}


$\mathrm{OH}$

3
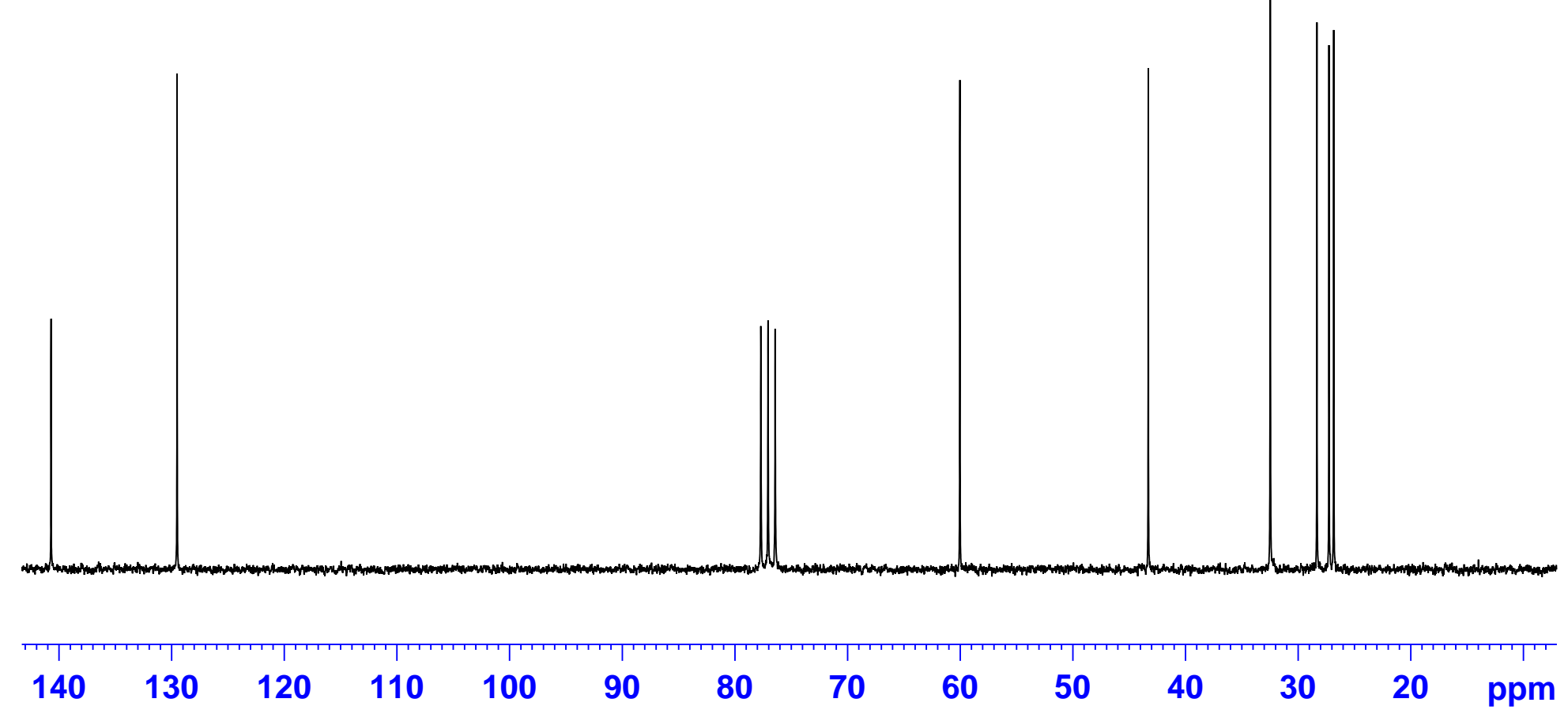


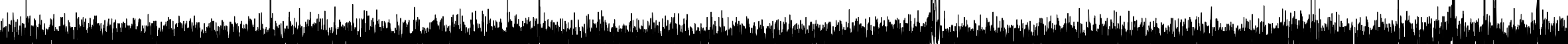

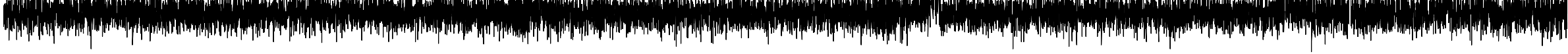


S

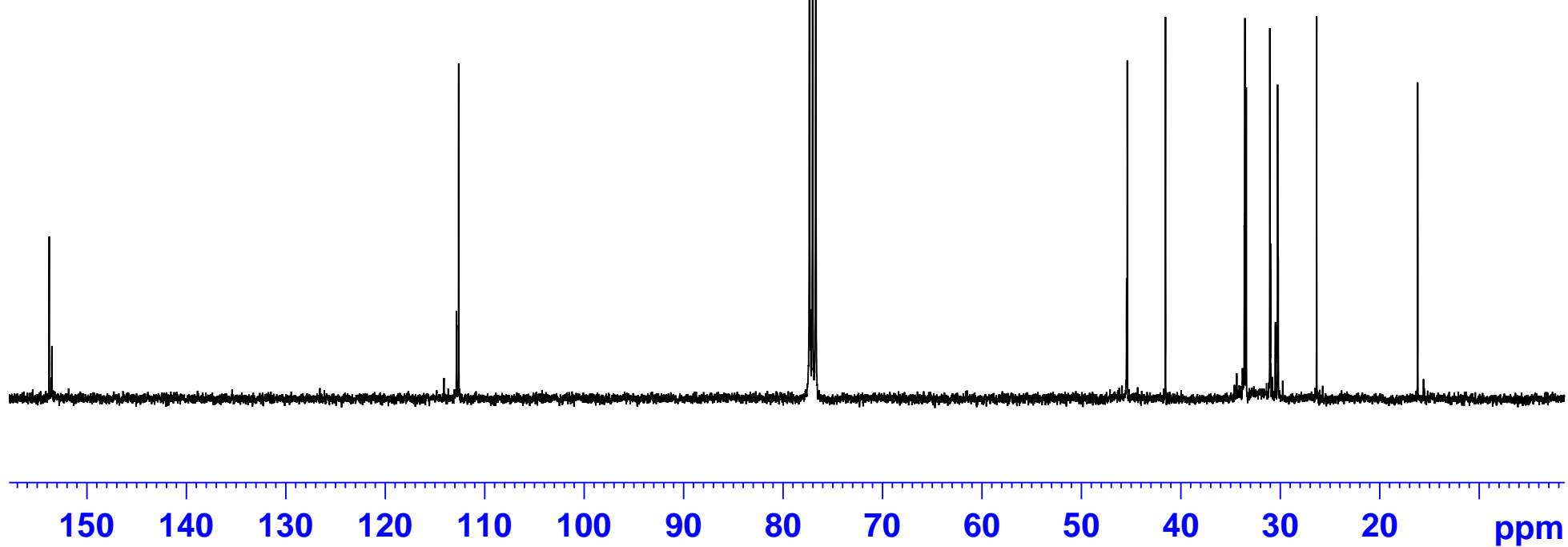




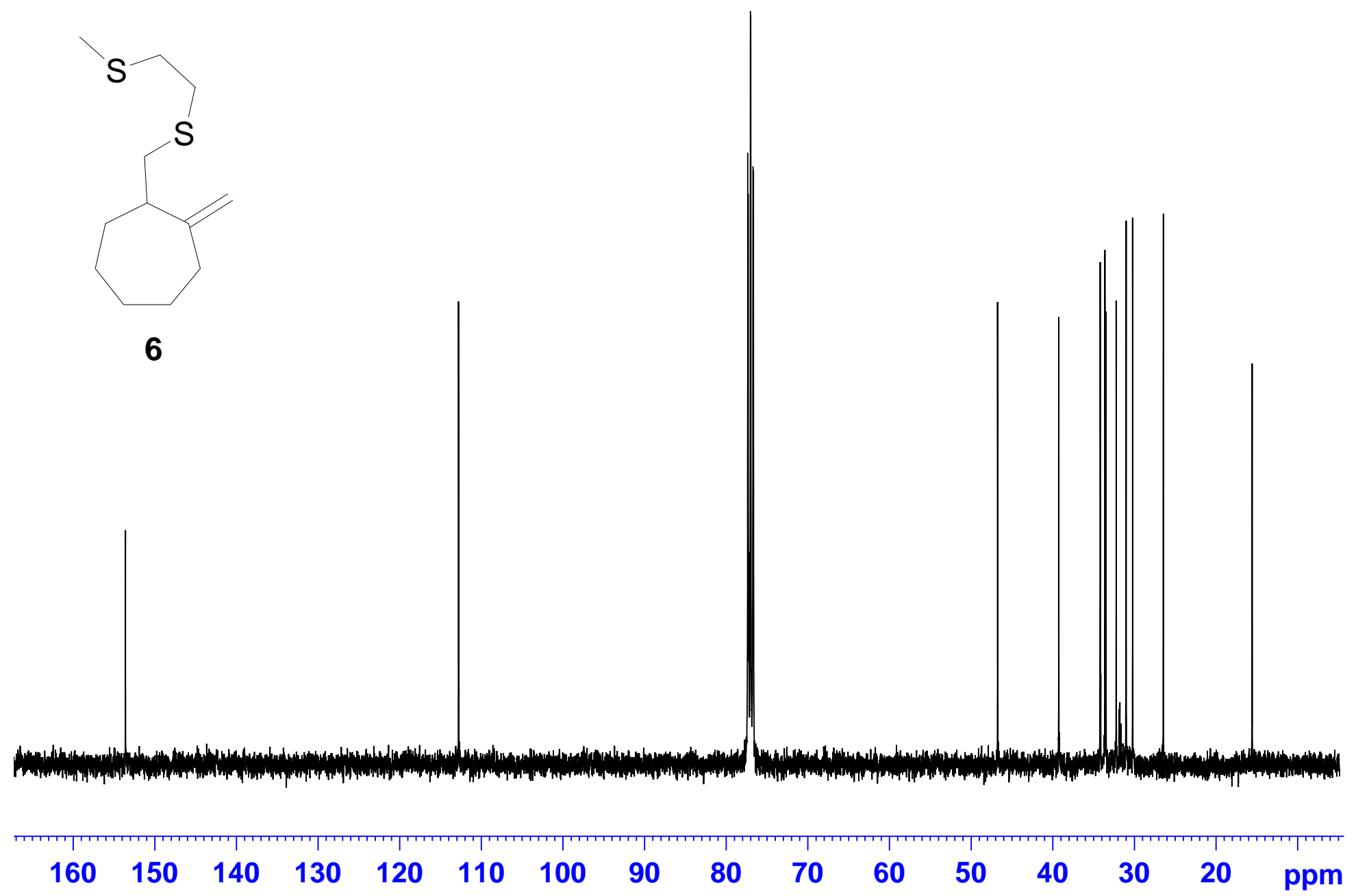


II

7
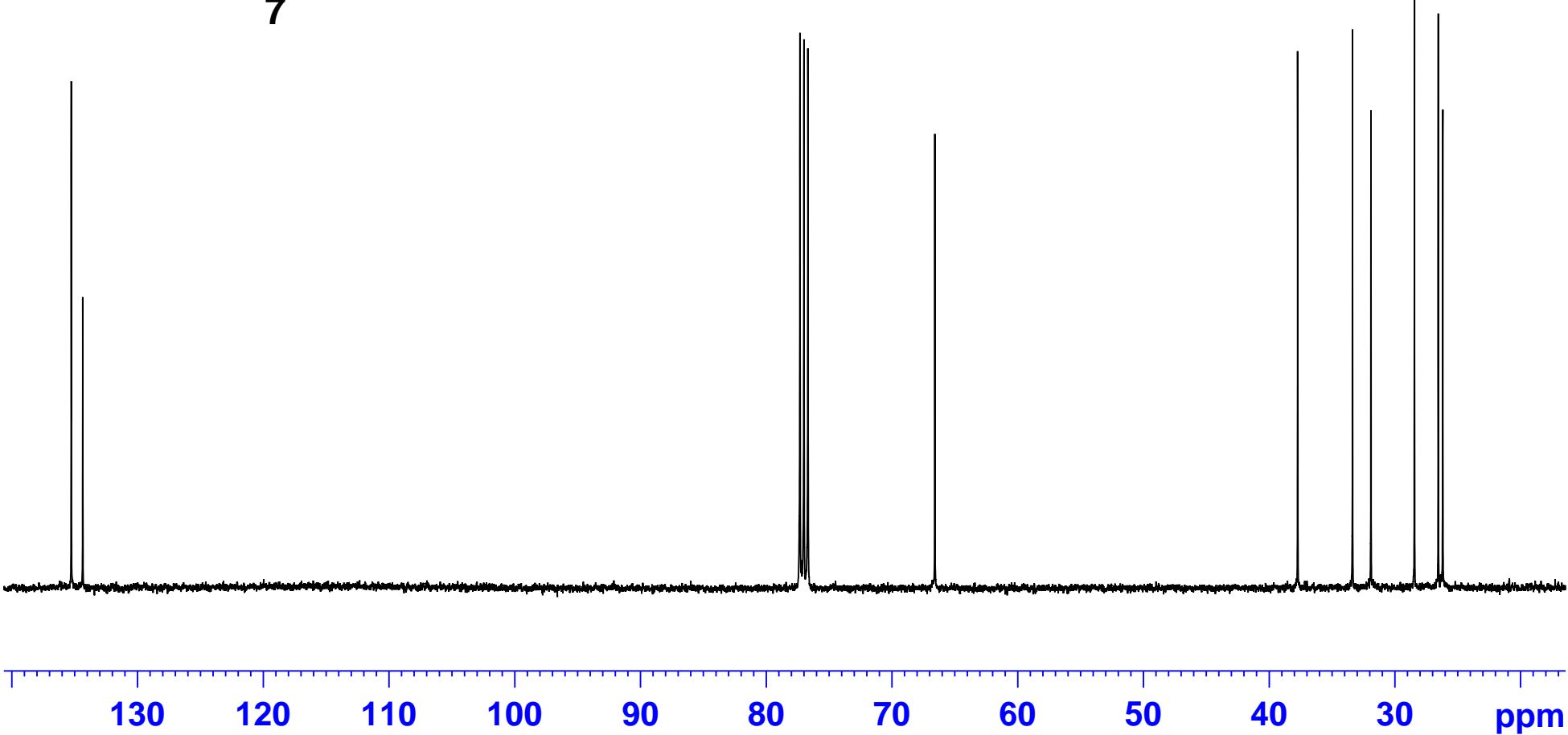


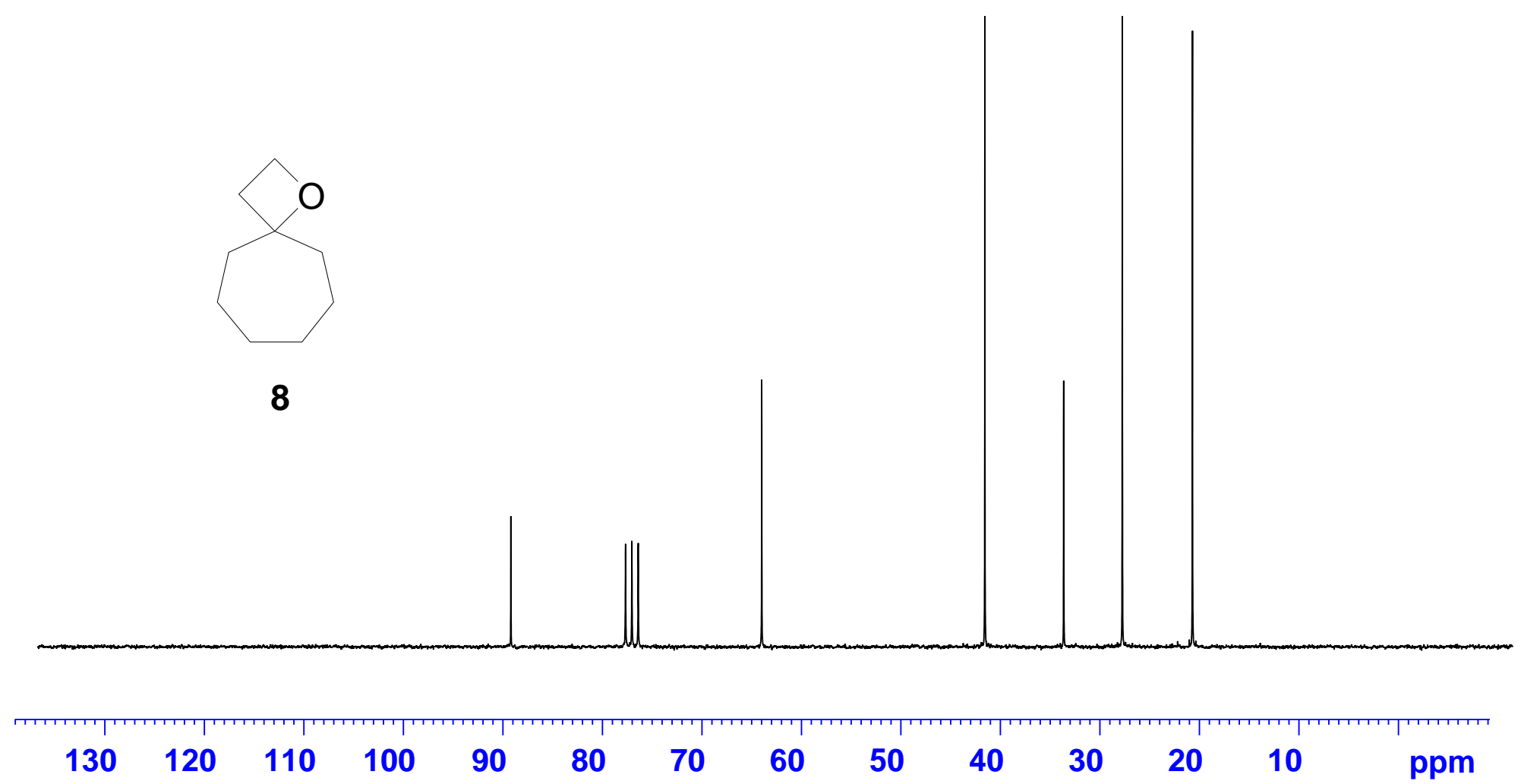


$\mathrm{NO}_{3}$

$\mathrm{HO} \mathrm{S}$

10
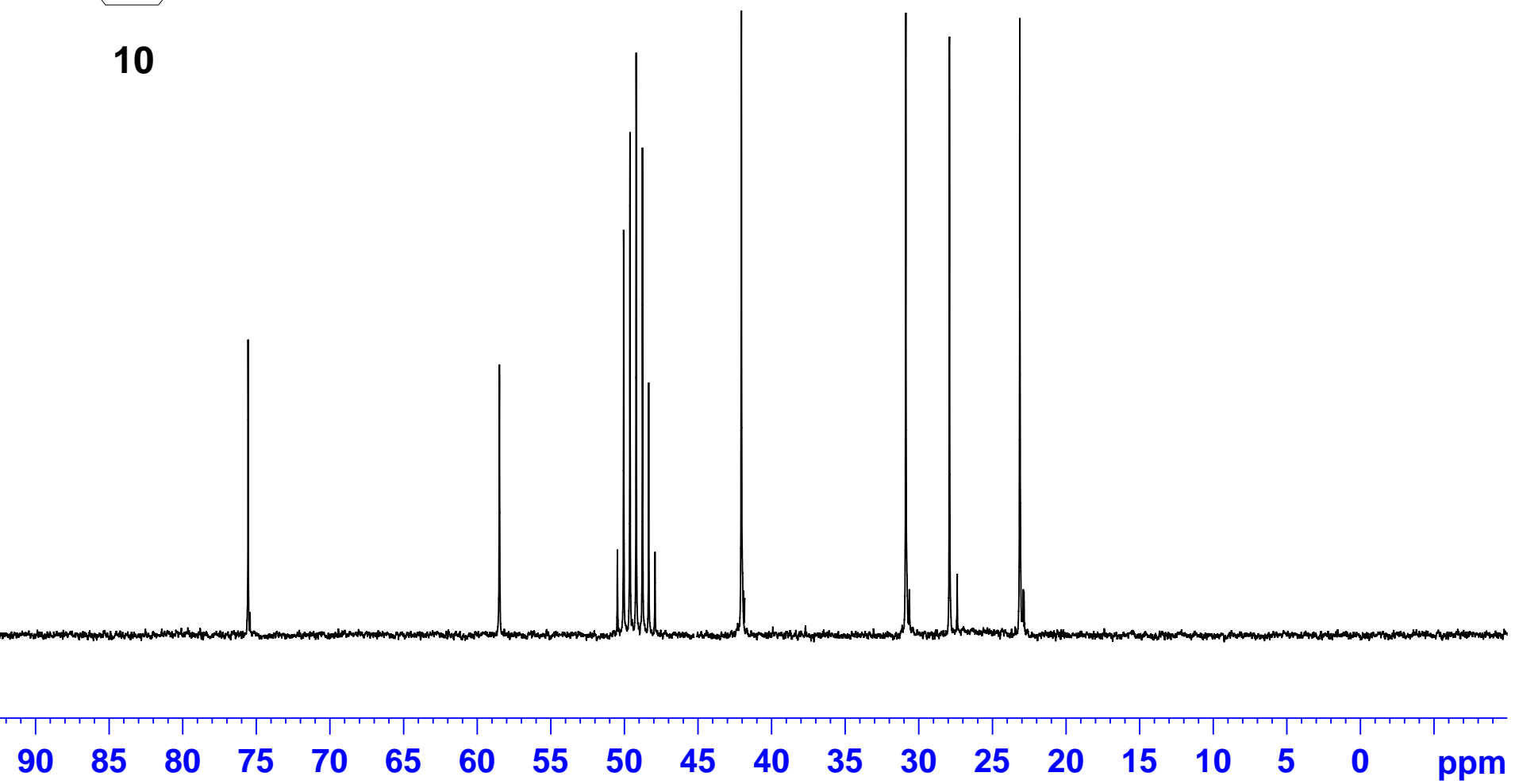


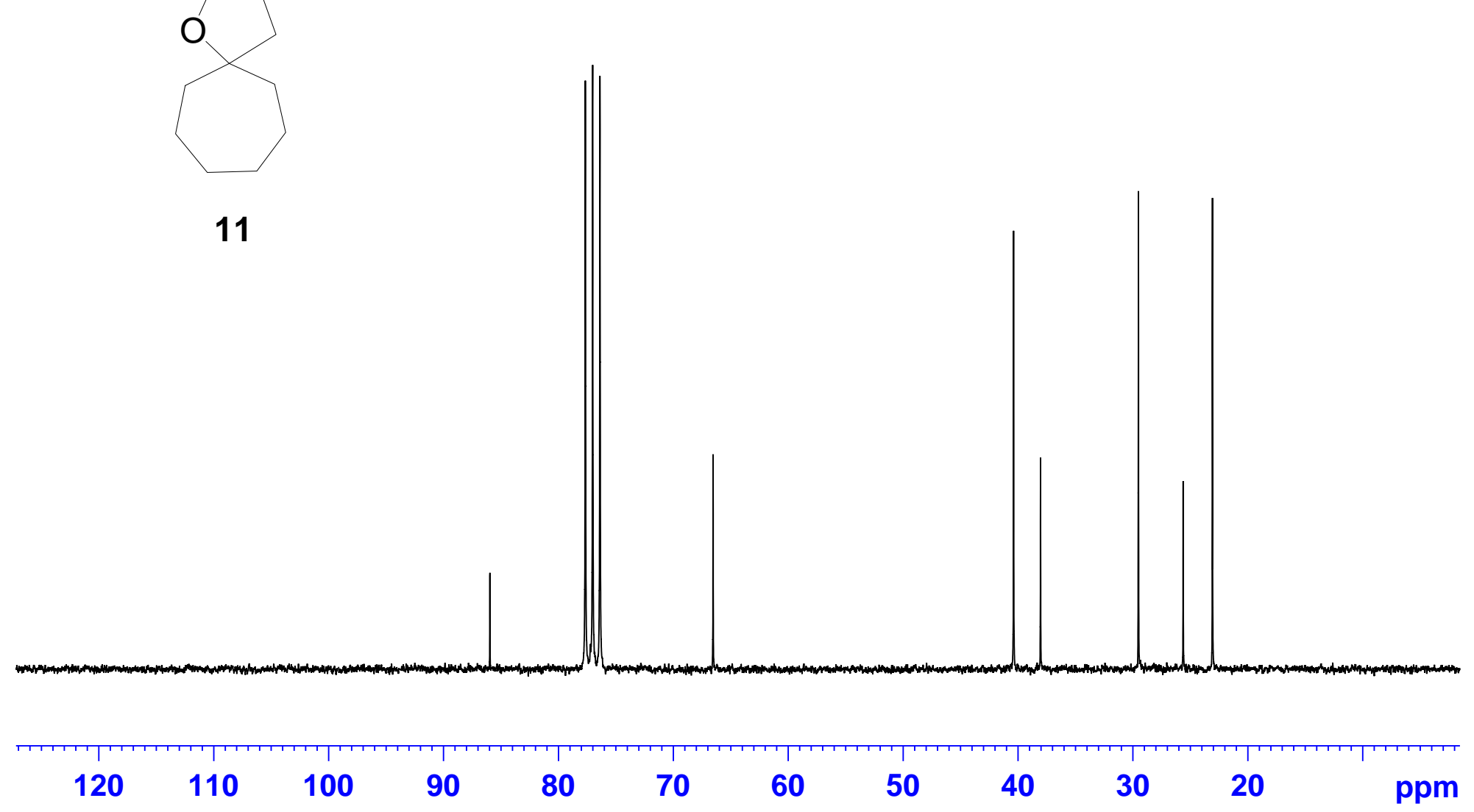


14

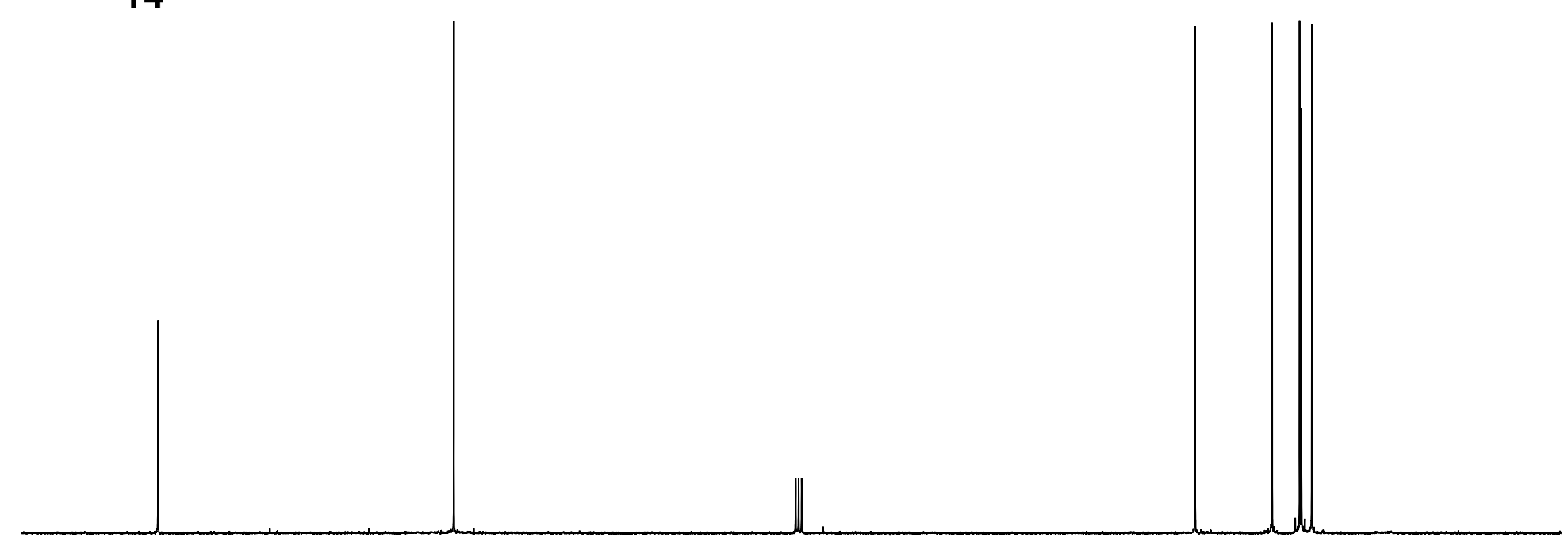

$\begin{array}{llllllllllllllll}150 & 140 & 130 & 120 & 110 & 100 & 90 & 80 & 70 & 60 & 50 & 40 & 30 & 20 & 10 & \text { ppm }\end{array}$ 


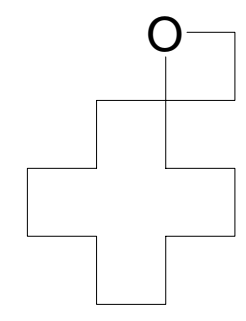

15

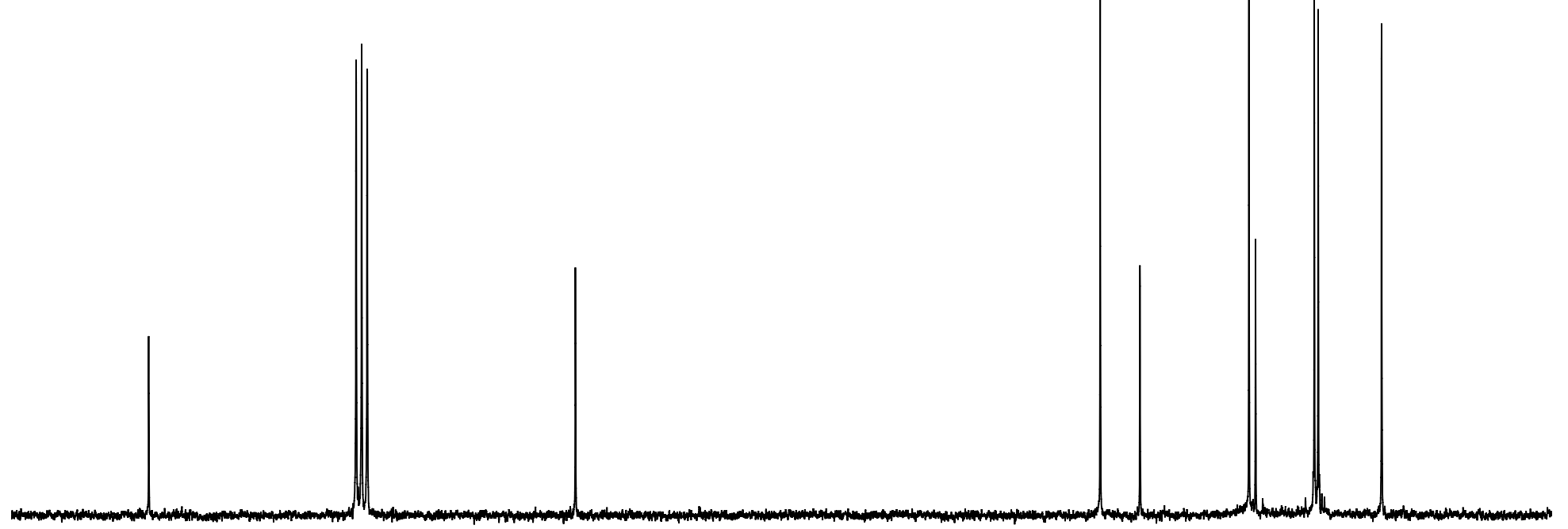

$\begin{array}{llllllllllllllllll}95 & 90 & 85 & 80 & 75 & 70 & 65 & 60 & 55 & 50 & 45 & 40 & 35 & 30 & 25 & 20 & 15 & \text { ppm }\end{array}$ 


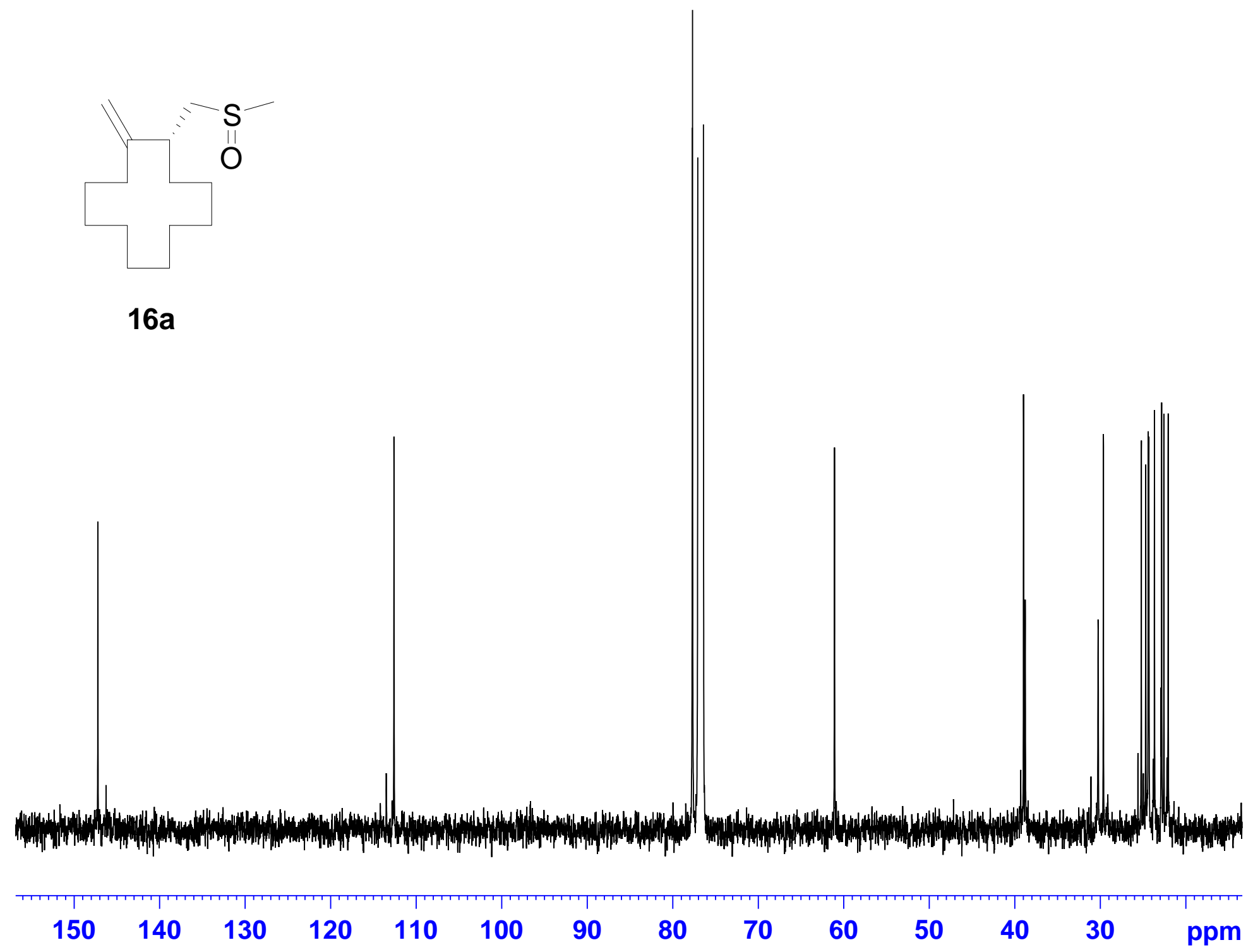




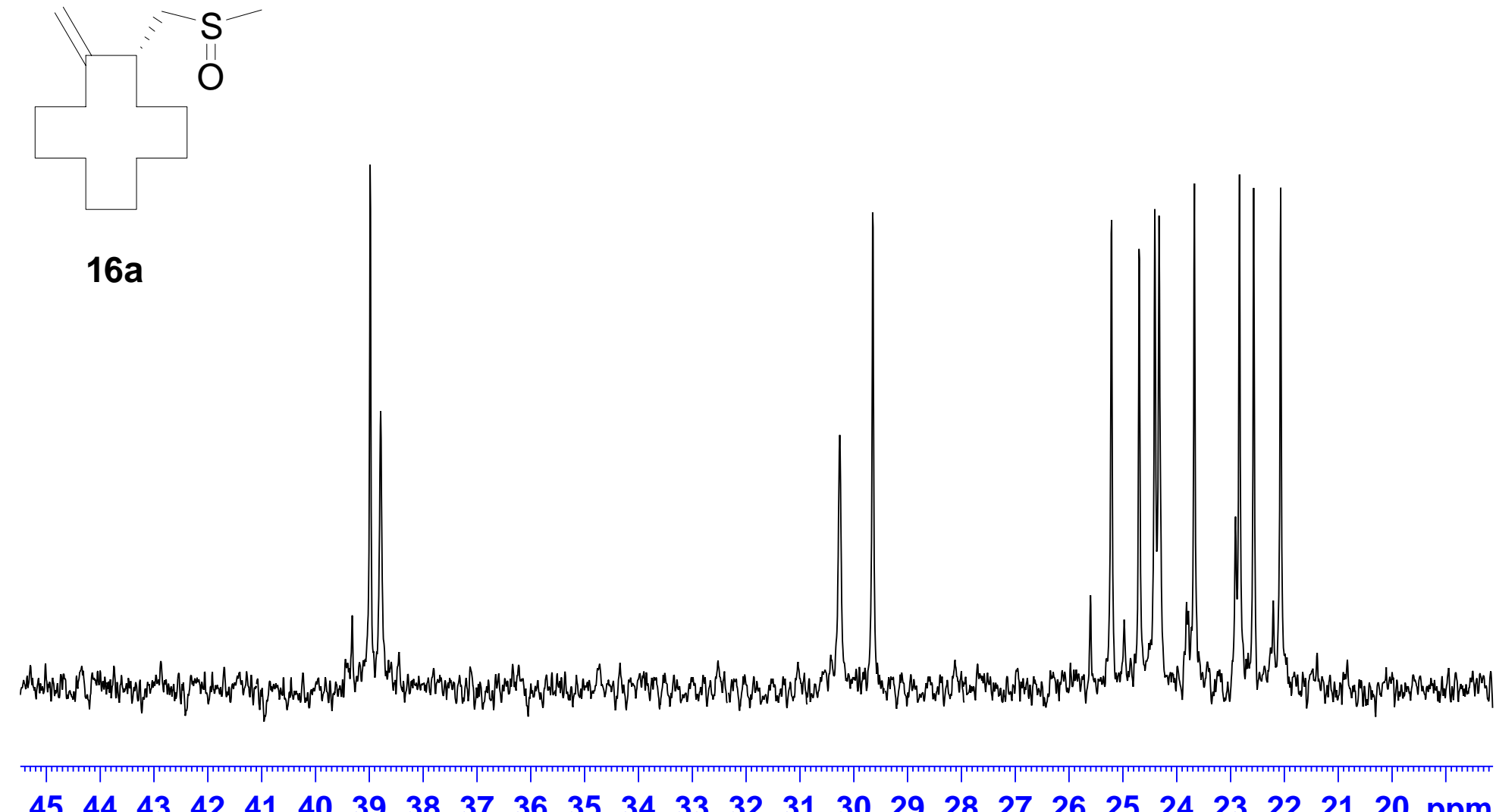

$\begin{array}{lllllllllllllllllllllllllll}45 & 44 & 43 & 42 & 41 & 40 & 39 & 38 & 37 & 36 & 35 & 34 & 33 & 32 & 31 & 30 & 29 & 28 & 27 & 26 & 25 & 24 & 23 & 22 & 21 & 20 & \text { ppm }\end{array}$ 
$/ \underset{1}{\mathrm{~S}}$

16b

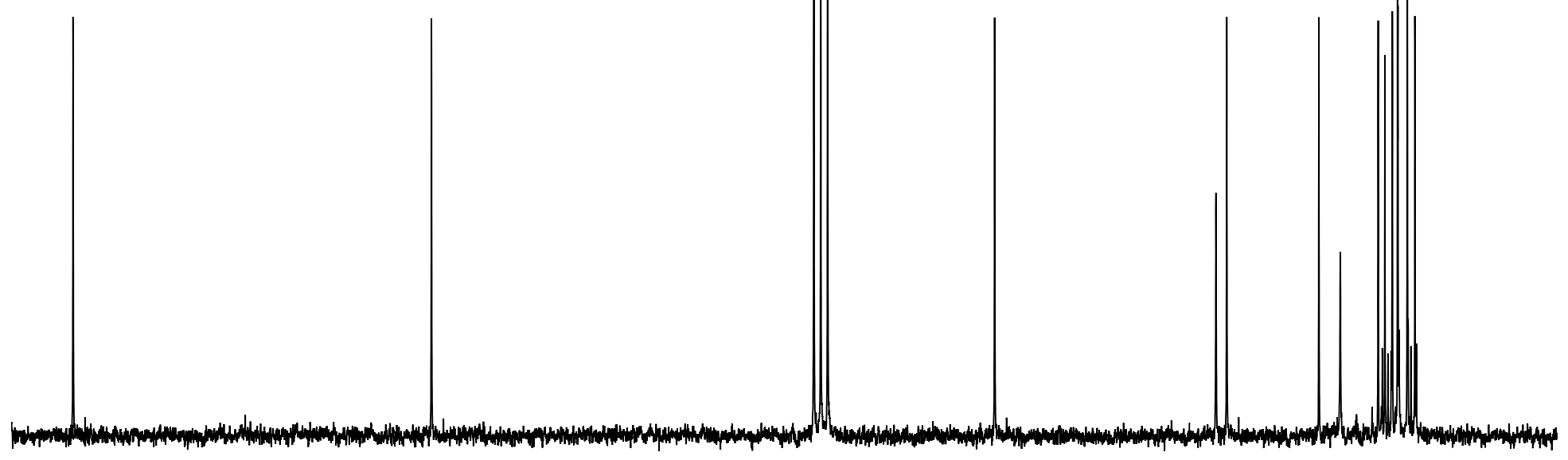

$\begin{array}{llllllllllllll}140 & 130 & 120 & 110 & 100 & 90 & 80 & 70 & 60 & 50 & 40 & 30 & 20 & \text { ppm }\end{array}$ 
S

17

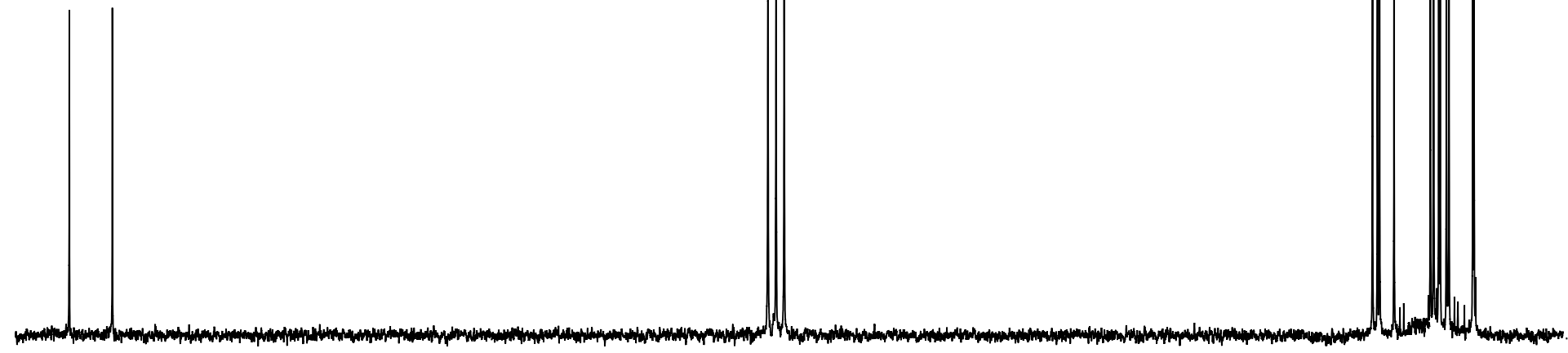

130

120

110

100

90

80

70

60

50

40

30

ppm 


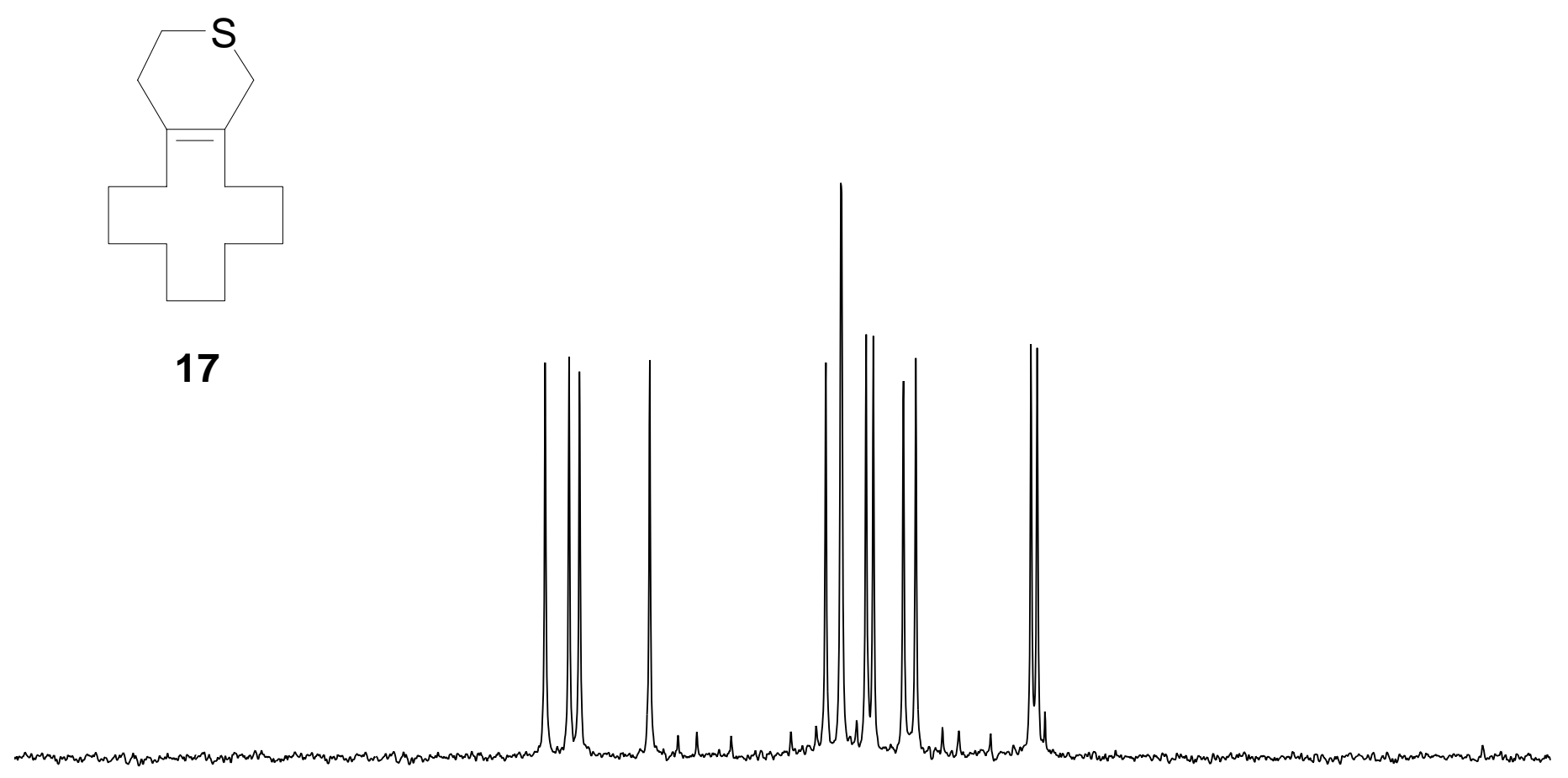

$\begin{array}{llllllllllllllllllllllllll}38 & 37 & 36 & 35 & 34 & 33 & 32 & 31 & 30 & 29 & 28 & 27 & 26 & 25 & 24 & 23 & 22 & 21 & 20 & 19 & 18 & 17 & 16 & 15 & \mathrm{ppm}\end{array}$ 


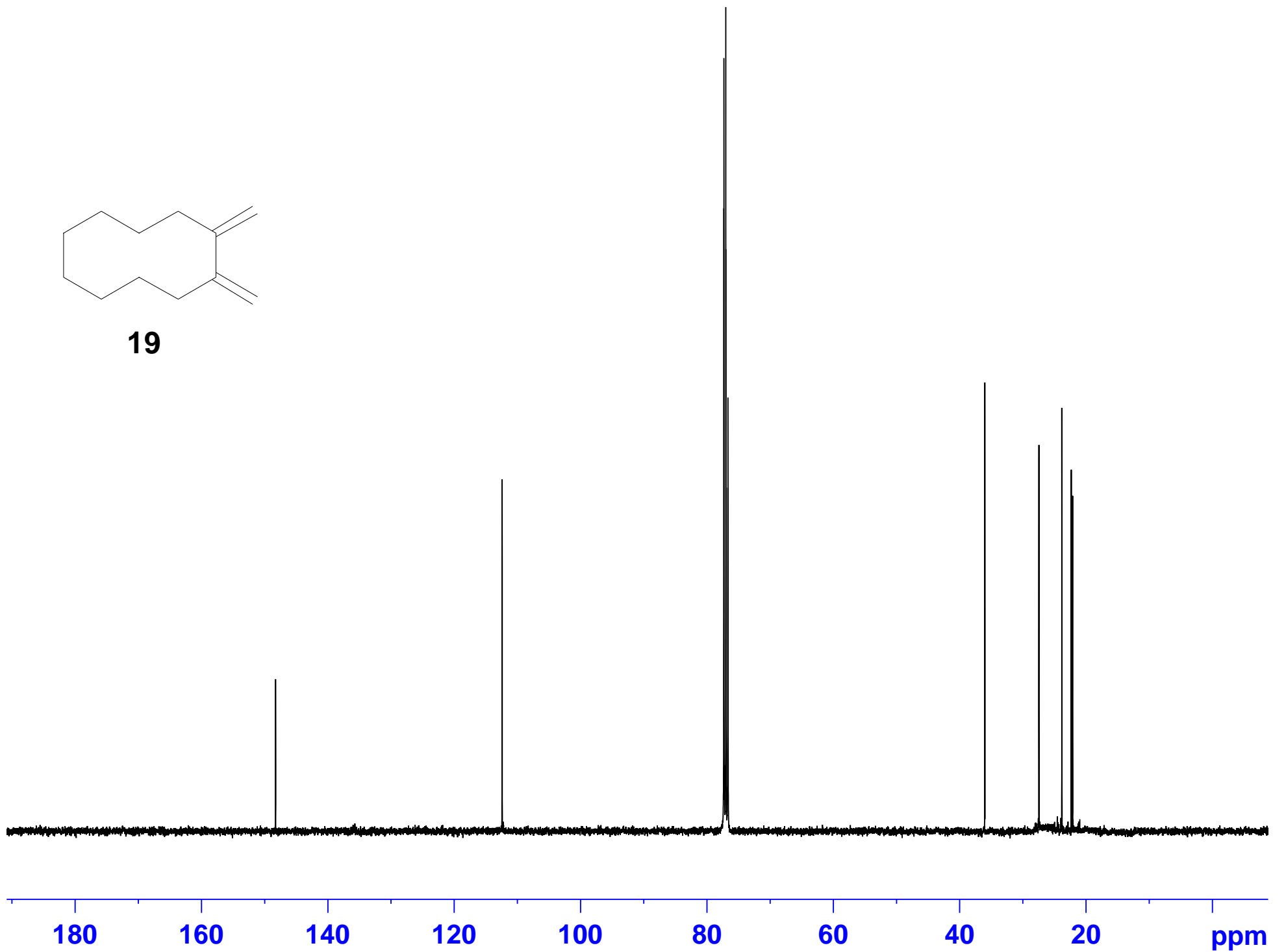

